\title{
Study of a Pneumatic Robot Utilizing a BTA (Bourdon Tube Actuator)
}

\author{
Yoshihide FUJIWARA, Yukio SAITO and Kazutaka HATTORI \\ Department of Industrial Mechanical Engineering Faculty of Science and Technology \\ Tokyo Denki University \\ Ishizaka, Hatoyama, Hiki-gun, Saitama 350-03 Japan
}

\begin{abstract}
The Pneumatic BTA (Bourdon Tube Actuator) is a stickslipless actuator which utilize a pair of sqiral bourdon tubes as a functional element and obtains displacement by air pressure. Compared with an electric motor and so on, the BTA is smaller and lighter; moreover, it generates a high torque and accurate positioning.

Before now it has been difficult for a cylindrical type pneumatic actuator to control accurate positioning because of sliding friction. Our laboratory developed a three-axis driving robot with 4-bar linkage utilizing a pneumatic BTA. We obtained ascillating of \pm 15 degrees and minimum sensitivity $0.6 \times 10^{-2}$ degree. A pneumatic BTA robot system makes possible minute action and accurate positioning.
\end{abstract}

\section{KEY WORD}

Torque and pasition controlled actuator, Pneumatic Robot, Stickslipless actuator

\section{Introduction}

BTA stands for "lourdon tube actuator," a rotary accillating actuator that uses spiral bourdon tube to convert air pressure into an angular diş̧lacement.

Among its features:

- It has a high weight torque ratio

- It has a small coefficient of friction, because structurally it has no sliding surface.

- It is leakness and is suitable for clean operation.

- It is capable of rotary ascillating operation at low speed without using a syeed reduction mechanism as in a geared motor.

Actuators are divided broadly into those that operate by energy conversion and thoe that operate by functional elements. A BTA has elements of both types, be- cause it converts pressure into angular (lisplacement, and the material and shape of its bourdon tules directly affect its characteristics.

Used in this studly was a spiral bourdon tube having a built-in air-pressure gauge.

We analyzed its pressure and displacement characteristics and derived calculation formulas that approximate its characteristics and are needed for BTA design. We also developed a positioning control system using this BTA that yields an angular molution $\left(0.6 \times 10^{-2} \mathrm{deg}\right)$ not available with conventional pneumatic actuators and a response of approximately $2.0 \mathrm{Iz}$.

In the laboratory we developed a pneumatic robot system using this actuator and built a three-axis driving robot. In this study we deacribe its functions and the system. 


\section{Basic Structure of The BTA ${ }^{1)}$}

In the BTA, two bourclon tubes positioning in the opposite direction, connected by a common rotation shaft. The inner end of each bourdon tube is connected to the common rotation shaft, and the outer end is anchored to the case. Pneumatic pressure is applied through the stationary part at the outer end. Here the pressure of the bourdon tubes is denoted by $\mathrm{P}_{\mathrm{A}}$ and $\mathrm{P}_{\mathrm{B}}$. With this simple structure, the rotation of the output rotation shaft is obtained from the pressure difference $\left(\mathrm{P}_{\mathrm{A}}-\mathrm{P}_{\mathrm{B}}\right)$ between the two bourdon tubes.

In a BTA, the characteristics of each individual unit vary slightly from the bourdon tube manufacturing process but they are averaged by being made to oppose each other as in Figure 1. The angular displacement of the BTA common rotation shaft is detected by a resolver, allowing the angular displacement to be determined.

\section{Calculation of Rotation Displacement Angle and Torque}

The Wolf theorem ${ }^{2}$ is used for calculating the rotation displacement angle and torque of a BTA. In a BTA, a pair of spiral bourdon tubes, which have a flat-oval crass-section, is used as the driving element, as in Figure 1. Denoting the supplied pressure by $\mathrm{P}_{\mathrm{A}}$ and $\mathrm{P}_{\mathrm{B}}$, the rotation displacement angle by $\theta_{\mathrm{BTA}}$, and the torque by $\mathrm{T}_{\mathrm{BTA}}$, according to the Wolf theorem the change in the bending radius $\Delta R_{A}$ and torque $T_{A}$ with respect to bourdon tube $A$ are given by

$$
\begin{aligned}
& \Delta R_{A}=\frac{a^{3} K_{3}}{E h^{3}\left(1+\lambda^{2} K_{1}\right)} P_{A} R \\
& T_{A}=\frac{a^{6} K_{4}}{R h^{2}\left(1+\lambda^{2} K_{2}\right)} P_{A}
\end{aligned}
$$

and the change in the bending radius $\Delta R_{B}$ and torque $\mathrm{T}_{\mathrm{B}}$ with respect to bourdon tube $\mathrm{B}$ are given by

$$
\begin{aligned}
& \Delta R_{B}=\frac{a^{3} K_{3}}{E h^{3}\left(1+\lambda^{2} K_{1}\right)} P_{B} R \\
& T_{B}=\frac{a^{6} K_{4}}{R h^{2}\left(1+\lambda^{2} K_{2}\right)} P_{B}
\end{aligned}
$$

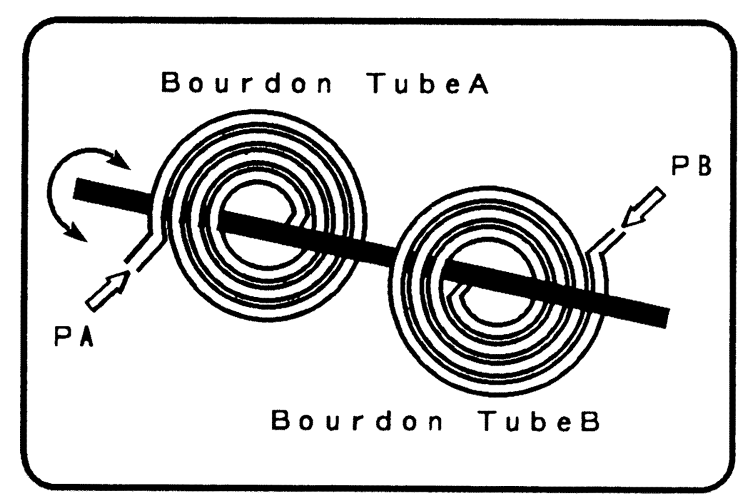

Fig. 1 Basic structure of BTA

Table. 1 Specifications of Bourdon Tube

\begin{tabular}{|c|c|}
\hline Material & SUS 316 TP \\
\hline $\begin{array}{c}\text { Shpe of } \\
\text { Cross Section }\end{array}$ & FLAT-OVAL \\
\hline $\begin{array}{c}\text { The Nimber of } \\
\text { Windings }\end{array}$ & 3.25 \\
\hline Pressure & $0 \sim 3(100 \%)\left(\mathrm{Kg} / \mathrm{cm}^{2}\right)$ \\
\hline $\begin{array}{c}\text { Displacement } \\
\text { of angle }\end{array}$ & $22.5(\mathrm{deg})(100 \%)$ \\
\hline
\end{tabular}

$\mathrm{K}_{1}, \mathrm{~K}_{2}, \mathrm{~K}_{3}$, and $\mathrm{K}_{4}$ are constants determined by the tube cross-section and its long-short radius ratio and are determined by approximating the flat-oval crose soction with an approximation ellipse. Theor onnstants are important in calculating the rotation disylacement angle and torque of a BTA. In the formulas here, the symbols of Figure 2 are as follows.

$\mathrm{E}$ : modulus of longitudinal elasticity

$\theta$ :angle between two crans-sections perpendicular of tube

$2 \mathrm{a} 2 \mathrm{~b}$ : Major or Minor axis of the cross-section in regard to a neutral surface of wall

$\lambda: \mathrm{a}^{2} \mathrm{Rh}$

$\Delta R$ : changing of radius after the pressum is put upon the tube

$\mathrm{h}$ : thickness of tube wall

T: torque

The amount of movement $S_{A}, S_{B}$ of the tip of each bourdon tube isexpresed as follows.

$$
\begin{aligned}
& S_{A}=\sqrt{2(1-\operatorname{Cos} \theta)-2 \theta \operatorname{Sin} \theta+\theta^{2}} \times R_{A}-(5) \\
& S_{B}=\sqrt{2(1-\operatorname{Cos} \theta)-2 \theta \operatorname{Sin} \theta+\theta^{2}} \times R_{B}-(6)
\end{aligned}
$$




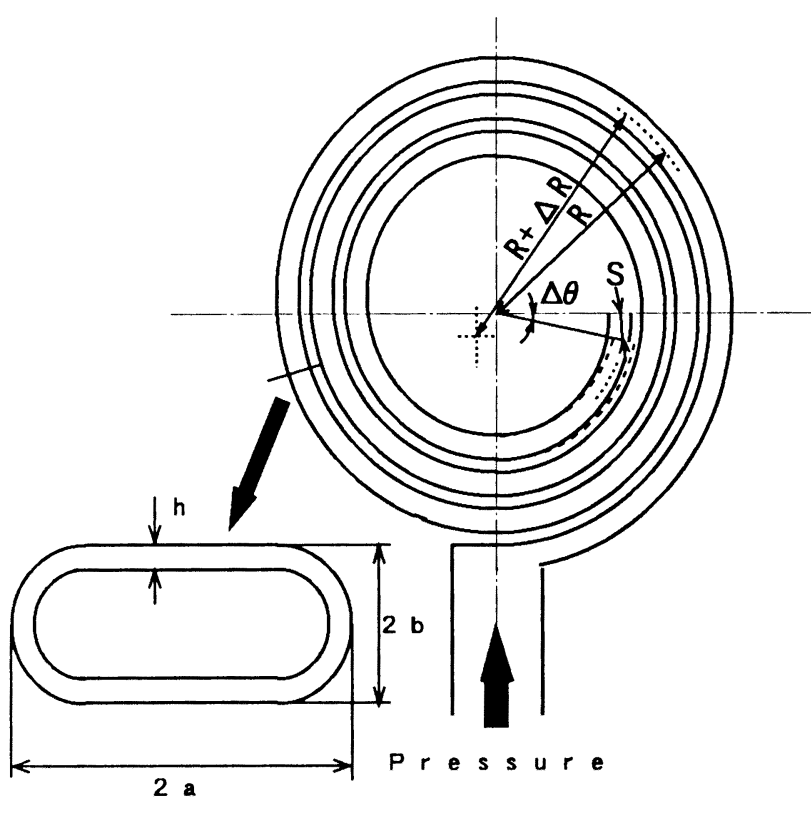

Fig. 2 BTA dimensions

The amount of movement $\mathrm{S}_{\mathrm{A}}, \mathrm{S}_{\mathrm{B}}$ of the tip of a bourdon tube is the amount of displacement of the bourdon tube that is connected to the rotation shaft of the BTA, $s 0 \mathrm{R}$ is constant, and converting $S_{A}, S_{B}$ to the rotation angle displacement $\theta_{\mathrm{A}}, \theta_{\mathrm{B}}$ (deg) of the bourdon tubes yields the following.

$$
\begin{aligned}
& \theta_{A}=\frac{360}{2 \pi R} \sqrt{2(1-\operatorname{Cos} \theta)-2 \theta \operatorname{Sin} \theta+\theta^{2}} \times \frac{a^{3} K_{3} P_{A} R}{E h^{3}\left(1+\lambda^{2} K_{1}\right)} \\
& \theta_{B}=\frac{360}{2 \pi R} \sqrt{2(1-\operatorname{Cos} \theta)-2 \theta \operatorname{Sin} \theta+\theta^{2}} \times \frac{a^{3} K_{3} P_{B} R}{E h^{3}\left(1+\lambda^{2} K_{1}\right)}
\end{aligned}
$$

From this the rotation displacement angle $\theta_{\mathrm{BTA}}$ of the BTA, taking the reaction of Bourdon tubes A and B into account, is

$$
\theta_{B T A}=\mu \times \frac{\theta_{A}-\theta_{B}}{2}
$$

where $\mu$ is a coefficient that depends on the material of the bourdon tubes and the precision of its machining. Similarly, the torque $\mathrm{T}_{\mathrm{BTA}}$ of the BTA is as follows.

$$
T_{B T A}=\frac{T_{A}+T_{B}}{2}\left(K g f^{\bullet} \mathrm{cm}\right)
$$

Figure 3 shows the output rotation torque $\mathrm{T}$ $(\mathrm{Kgf} \cdot \mathrm{cm})$ with respect to the rotation disy)lacement angle $\alpha$ of the BTA. The torque varies linearly with changes in the rotation displacement angle $\alpha$ (deg) Figure 4 shows, for bourdon tubes $\mathrm{A}$ and $\mathrm{B}$, the relationship between the pressure $\mathrm{P}\left(\mathrm{Kgf} / \mathrm{cm}^{2}\right)$ and rotation displacement angle $\alpha$ and the set pressure $\mathrm{P}_{1}$ with respect to the applied pressure $\mathrm{P}_{2}$, as measured optically using laser spot triangulation. For the theoretical value in the diagram, the above-determined theoretical formula (9) is used, and coefficient $\mu$ is 1 . In this diagram too, the angle varies linearly with the pressure.

\section{Structure of BTA Robot}

We built in laboratory a three-axis driving robot using three of these BTAs. The purpore is cause letters to be written on minute particles as the first step. For this purpose, a four-bar linkage is adopted, and the hand part is a mechanism that always moves parallel to the X-Y plane.

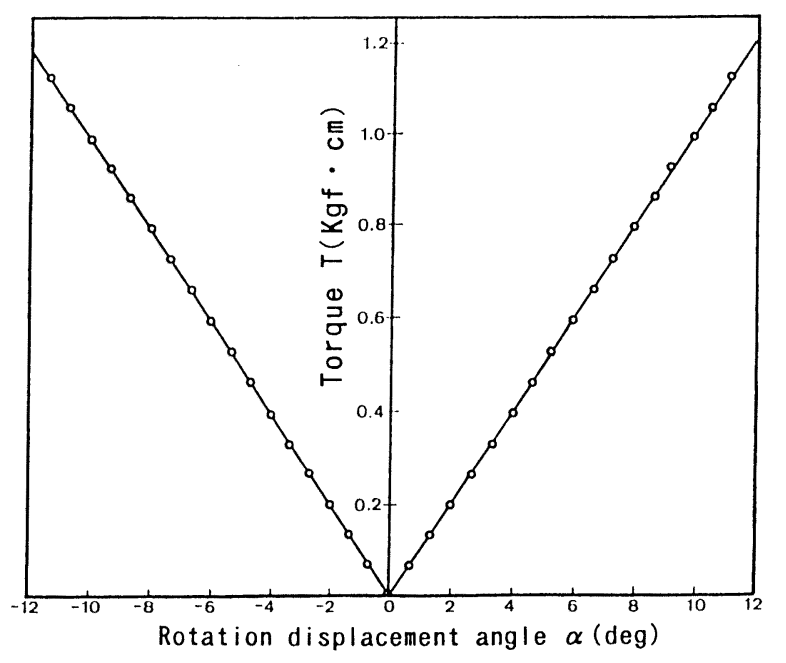

Fig.3 Characteristics angle / torque

In Y-direction changes with respect to a conorlinate system as shown in the diagram, displacement is obtained by driving the Y-axis BTA, and the hand part is moved from $\mathrm{P}$ to $\mathrm{P}^{\prime}$ parallel to the $\mathrm{X}-\mathrm{Y}$ plane by making $\mathrm{ABCD}$ in Figure 5 (a) into a parallelogram. Similarly for changes in the X direction, displacement is obtained by driving the $\mathrm{X}$-axis BTA with respect to coordinate system as shown in Figure 5(a), and by making EFGH in Figure 5 (b) into a parallelogram, the hand part is moved parallel from $\mathrm{P}$ to 
$\mathrm{P}$. For changes in $\mathrm{Z}$ direction, that is, changes in the height of the hand part, the hand tip part is displaced from $\mathrm{P}$ to $\mathrm{P}^{x}$ by driving the two-axis BTA. Denoting the angle of the respective BTAs by $\theta_{\mathrm{X}}, \theta_{\mathrm{Y}}, \theta_{\mathrm{Z}}$, this is expressed as follows.

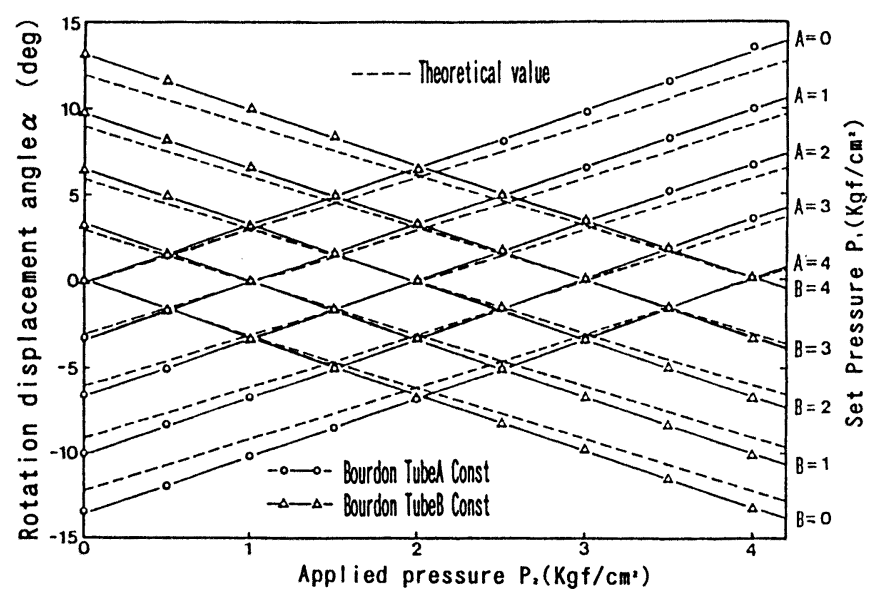

Fig. 4 Static characteristics of rotational angle and pressure

$$
\begin{aligned}
& \theta_{X}=\operatorname{SIN}^{-1} \frac{X-d X}{L \operatorname{COS} \theta_{Y}} \\
& \theta_{Y}=\operatorname{SIN}^{-1} \frac{Y}{L} \\
& d X=M-M \operatorname{CoS} \theta_{2}
\end{aligned}
$$

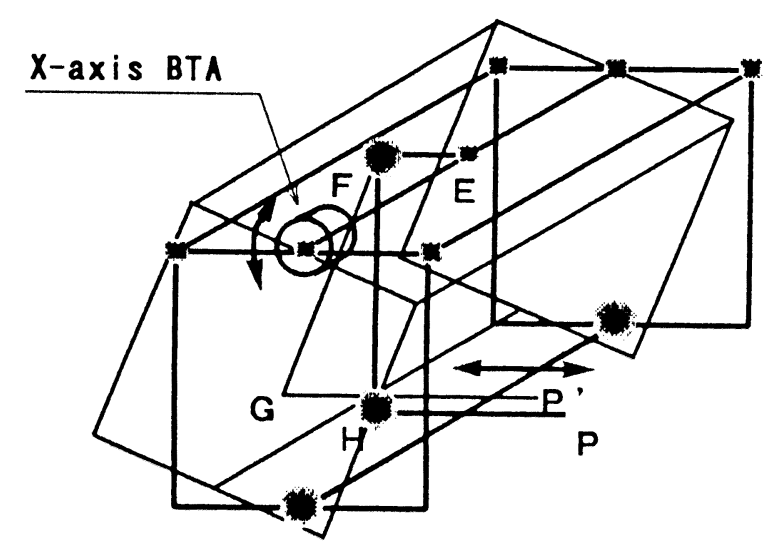

\section{BTA Robot System}

(b) X Direction

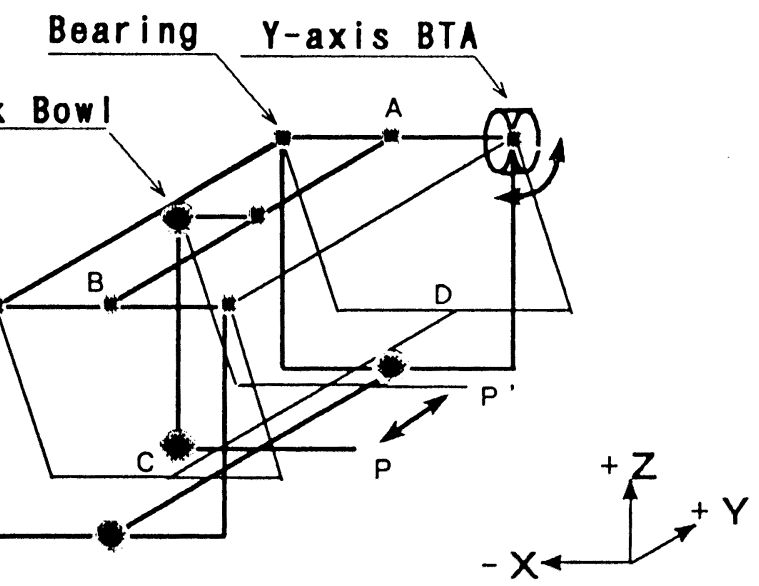

(a) Y Direction

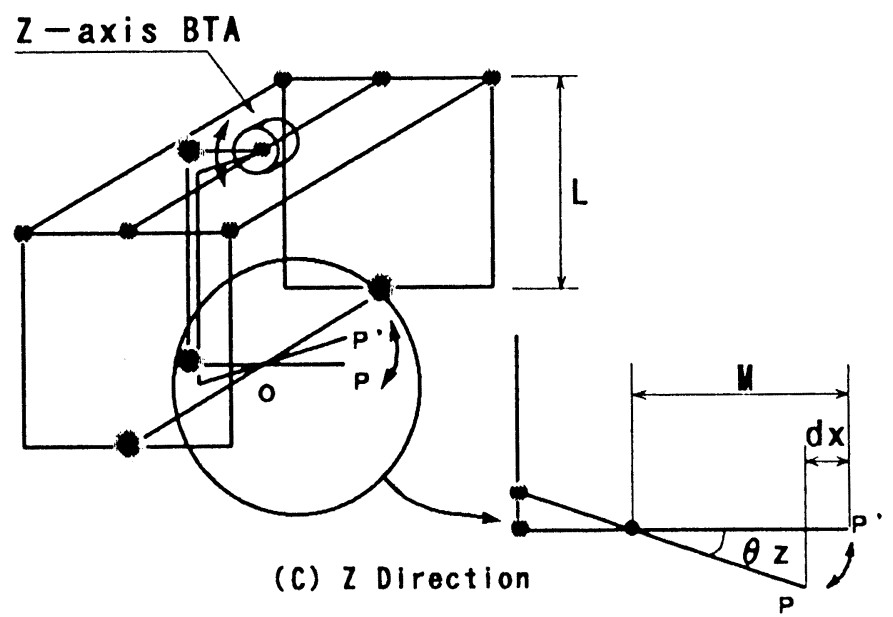

Fig. 5 Mechanisms of BTA robot

Figure 6 is appearance of the BTA robot, Figure 7 is its block diagram. The BTA robot control system includes a host computer(PC9821), RD converter, compressor, electric-air regulator, the robot itself, a resolver, a dryer, and a lubricator. A 12-bitD/A converter board and 8255 board are built into the expansion slots of the host computer, the pressure signal to the electric-air regulators D/A converted, the actually obtained rotation angle is detected by the brushless resolver, RD conversion is performed, and it is fed into the computer. The actually obtained rotation angle displacement $\phi_{\mathrm{i}}$ iscalculated by RD-converting the resolver signal, and it is compared with the input displacement angle $\theta_{\mathrm{S}}$. Then feedback is done so that $\phi_{\mathrm{i}}=\theta_{\mathrm{S}}$ 


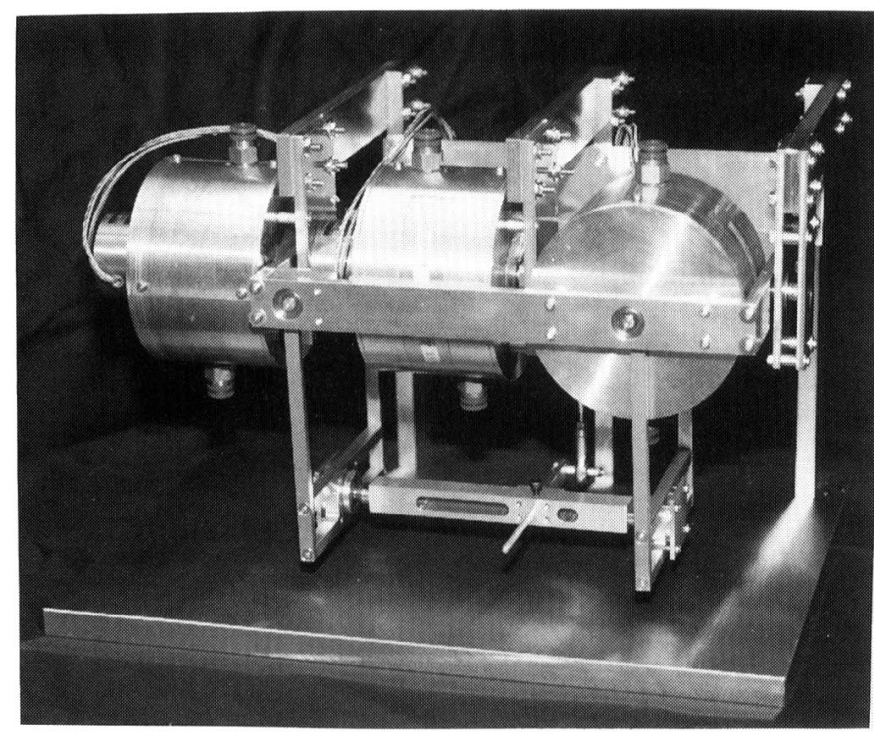

Fig. 6 Appearance of BTA robot

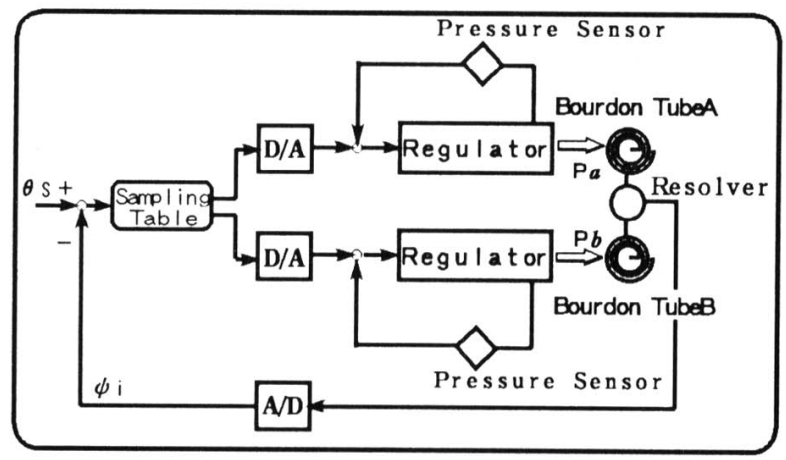

Fig. 7 Block diagram of BTA control system

The output signal of the personal computer can be output with a resolution of $1.22 \times 10^{-3}$, so the output then can be controlled with a resolution of $1.22 \times 10^{-3}$ $\left(\mathrm{Kgf} / \mathrm{cm}{ }^{2}\right)$. Actually it is constrained by the resolution of the electric-air regulator and is $0.6 \times 10^{-3}\left(\mathrm{Kgf} / \mathrm{cm}^{2}\right.$ ). Figure 8 presents the frequency response of the BTA control system. It shows that it can track closely up to about $2 \mathrm{~Hz}$. The output control valve used in this system is an off-the-shelf electric-air regulator. Since the frequency response of this electric-air regulator is $2 \mathrm{~Hz}$, the frequency response of BTA control system depends to a great extent on this electric-air regulator. Because of this an improvement in the dynamic characteristics of the electricair regulator improves the dynamic characteristics of the BTA control system.

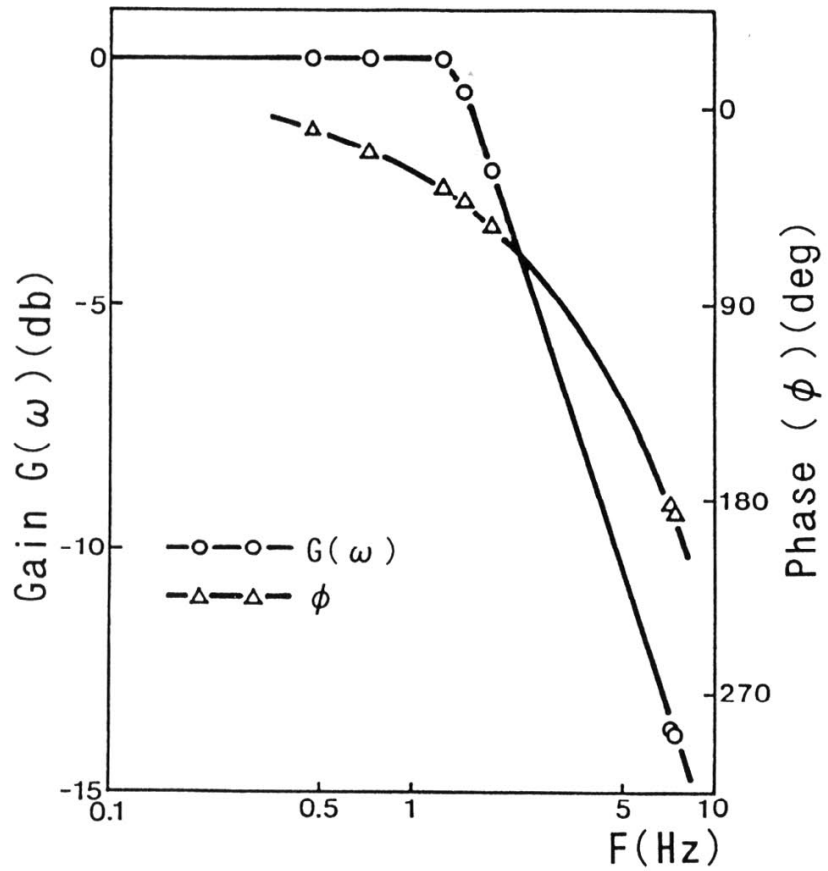

Fig. 8 Bode diagram of BTA control system

\section{Relationship of Tip Displacement and Pressure}

Figure 9 shows the result of measuring with a cathetometer the movement of the BT'A robot hand's tip) end when the pressure is varied from 0 to $5\left(\mathrm{Kgf} / \mathrm{cm}^{2}\right)$ based on the coordinate system of Figure 5. When this is done the pressure of the other bourdon tube is kejt constant at $0\left(\mathrm{Kgf}_{\mathrm{cm}}{ }^{2}\right)$. On each axis the amount of movement is symmetrical in the positive and negative directions a nonlinear curve described, and hysteresis is observed. The reason for this is thought to be that because (1) applied BTA of measuring Bourdon Tube could not obtain the torque for the purpose and (2) the bourlon tube is an elastic body, insufficient rigidity is obtained at pressure of $0-5\left(\mathrm{Kgf}_{\mathrm{cm}}{ }^{2}\right)$, so because of the structure of the BTA robot there are interactions with the weight of the link parts, directly or by friction. The results shown in Figure 4 are for a single BTA, no load is applied to the rotation shaft, and the changes linear, but firm these results too it can be inferned that the weight of the link parts and friction play a large role. 

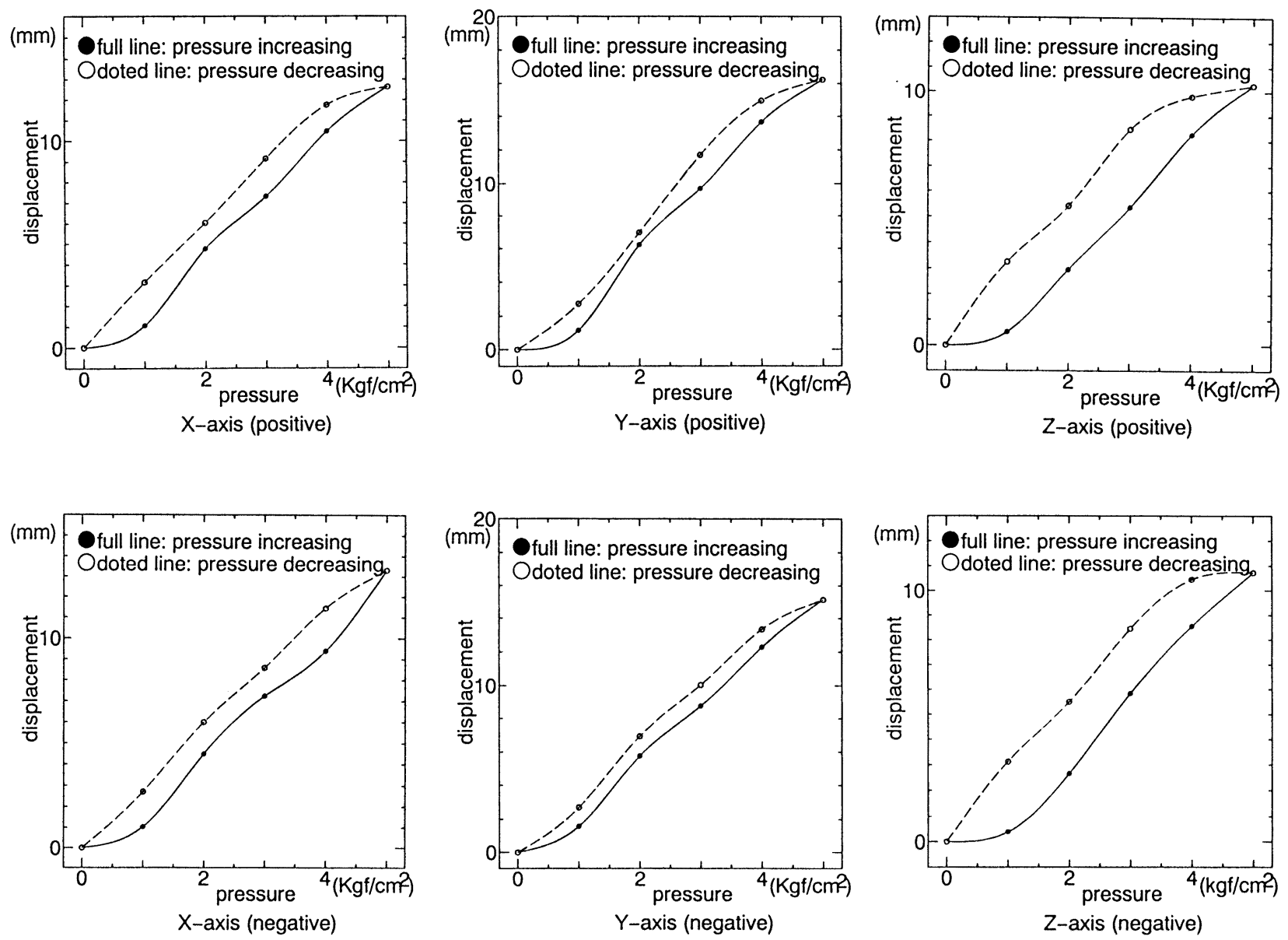

Fig. 9 Displacement-pressure curves for the end effector of robotics

Near $0\left(\mathrm{Kgftcm}^{2}\right)$ there is a big difference in the amount of movement between the case when pressure is reduced. This difference is attributed to the fact that a certain pressure is needed for operating the exhaust valve in the back pressure chamber of the solenoid nozzle, and when the pressure increases, the pressure needed for operating this is near $0\left(\mathrm{Kgf}_{\mathrm{gm}}{ }^{2}\right)$, and this effect becomes apparent.

\section{Conchusion}

To compensate for the characteristics of compression fluid, which are a drawback of pneumatic actuators, it is possible to take the average of two bourdon tubes by opposing them to each other. Because there is no stickslip, there is no need to use lubrication oil, and because a
BTA angle resolution of $0.6 \times 10^{-2}$ degree is obtained, improvement in the dynamic characteristics of electric-air regulator can be expected, as well as three-axis driving robots made for the purpose of fine operations such as in bio-technology, based on use at higher pressure.

\section{Reference}

1) Yoshihide.F, Yukio. S and Kazutaka. H, Study of a Pneumatic Robot Utilizing a BTA, Japanese Council or IFToMM (First Symposium), pp1-6, (1994)

2) Alfred Wolf, Tuisa, Okla, An Elementary Theory of ourdon Gauge, Journal of $A$ pplied Mechanics vol.13, no.3. pp209-210f 